\title{
Newtonian and modified newtonian gravitational simulation of spiral galaxies
}

\author{
Loay K. Abood ${ }^{1}$, Bushra A. Ahmed ${ }^{2}$, Mohammed A.Saleh ${ }^{3}$ \\ ${ }^{1}$ Department of Computer Science, College of Science, University of Baghdad, Iraq \\ ${ }^{2}$ Department of Astronomy and Space, College of Science, University of Baghdad, Iraq \\ ${ }^{3}$ Center of Renewable Energy, Ministry of Electricity, Baghdad, Iraq \\ E-mail: b_ali_ahmed@yahoo.com
}

\begin{abstract}
One of the most powerful tools for any stellar dynamics is the $\mathrm{N}$ body simulation. In an $N$-body simulation the motion of $N$ particles is followed under their mutual gravitational attraction. In this paper the gravitational $N$-body simulation is described to investigate Newtonian and non- Newtonian (modified Newtonian dynamics) interaction between the stars of spiral galaxies. It is shown that standard Newtonian interaction requires dark matter to produce the flat rotational curves of the systems under consideration, while modified Newtonian dynamics (MOND) theorem provides a flat rotational curve and gives a good agreement with the observed rotation curve; MOND was tested as an alternative to the dark matter hypothesis. So that MOND hypothesis has generated better rotation curves than Newtonian theorem.
\end{abstract}

Key words

Galaxies, kinematics and dynamics - Galaxies, spiral.

\section{Article info}

Received: Oct. 2012

Accepted: Apr. 2013

Published: Sep. 2013

$$
\begin{aligned}
& \text { محاكاة الجاذبية النيوتونية والنيوتونية المعلة للمجرات الحلزونية } \\
& \text { لؤي كاظم عبود1، بشرى علي احمد2، محمد احمد صالح³ }
\end{aligned}
$$

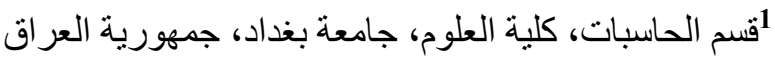

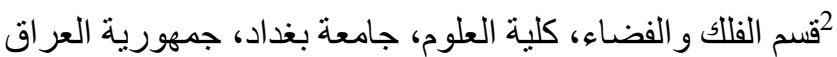

$$
\begin{aligned}
& \text { 3مركز الطاقات المتجددة، وزارة الكهرباء، جمهورية العراق اقلئاه }
\end{aligned}
$$

الخلاصة

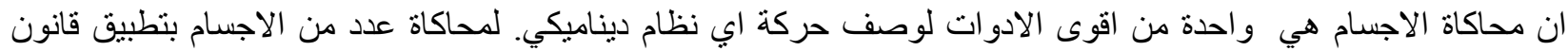

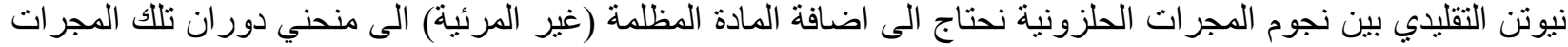

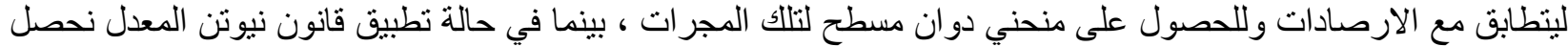

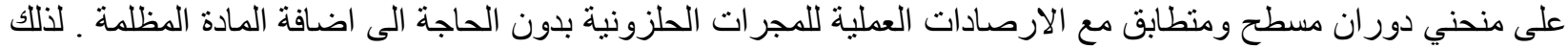

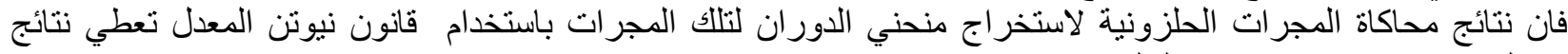
افضل من استخدام قانون نيوتن التقليدي.

\section{Introduction}

N-body numerical simulation has nowadays evolved into a widely used tool in cosmology thanks to the rapidly growing computer performance and, at the same time, to the development of more sophisticated numerical algorithms. An Nbody simulation numerically approximates 
the evolution of a system of bodies in which each body continuously interacts with every other body. A familiar example is an astrophysical simulation in which each body represents a galaxy or an individual star, and the bodies attract each other through the gravitational force. [1].

The parallelization of $N$-body algorithms along with specialized hardware has increased the number of particles in simulation to billions. There are now many parallelized $N$-body codes based on different algorithms including tree codes by Springel et al in 2001 and by Wadsley et. al. in 2004, PM methods along with new PM-tree hybrids by Dubinski et. al. in 2004 and by Springel in 2005. [2,3,4,5].

Some studies have addressed the question of the orbital structure in $N$-body systems of barred galaxies by Shen and Sellwood in 2004 and Ceverino and Klypin in 2007 in which, by definition, the orbits support the system self-consistently[6,7]. The study of the role of the chaotic orbits in the spiral structure was pursued by Voglis et. al. in 2006, using self-consistent $N$-body simulation of barred galaxies [8].

Spiral galaxies have most of their stellar mass in a large rotating disk, and only a modest fraction in a central spheroidal bulge. M. Martig and F. Bournaud in 2010 proposed that the continuous return of gas by stellar populations over cosmic times could help to solve this issue. A population of stars formed at a given instant typically returns half of its initial mass in the form of gas over 10 billion years, and the process is not dominated by supernovae explosions but by the long-term mass-loss from low-and intermediate-mass stars [9].

In the present work the Newtonian interaction is replaced by non-Newtonian interaction between the stars of a system that could explain the flat rotational curves which are characteristic of actual isolated spiral galaxies.

\section{Theory of the N-Body Problem}

In this section, the mathematical description of the gravitation will be given according to different hypothesis (Newtonian and modified Newtonian dynamics (MOND)); which is referred to Nbody problem simulation.

\section{The gravitational force for N-body problem}

Mathematically the N-body problem is formulated by the system of Ordinary Differential Equations (ODE) coming from Newton's laws of motion expressed as [10]

$$
\mathrm{m}_{\mathrm{i}} \frac{\mathrm{d} \overrightarrow{\mathrm{v}}_{\mathrm{i}}}{\mathrm{dt}}=\sum_{\substack{\mathrm{j}=1 \\ \mathrm{j} \neq \mathrm{i}}}^{\mathrm{N}} \overrightarrow{\mathrm{F}}_{\mathrm{ij}}
$$

where $m_{i}$ and $\vec{v}_{i}$ are the mass and velocity of the i-th particle, respectively, and $\mathrm{i}=1,2, \ldots, \mathrm{N}$. The force $\overrightarrow{\mathrm{F}}_{\mathrm{ij}}$ is usually the sum of external forces

$$
\overrightarrow{\mathrm{F}}(\overrightarrow{\mathrm{r}})=-\mathrm{G} \sum_{i=1}^{\mathrm{n}} \frac{\mathrm{Mm}_{i}}{\left|\overrightarrow{\mathrm{r}}_{i}-\overrightarrow{\mathrm{r}}\right|^{3}}\left(\vec{r}_{i}-\overrightarrow{\mathrm{r}}\right)
$$

$\overrightarrow{\mathbf{r}}_{i}$ represents the position vector.

When dealing with systems of stars or stellar systems, Newton's gravitational force will be used

$\frac{\mathrm{d} \vec{v}_{i}}{\mathrm{dt}}=\mathrm{G} \sum_{\substack{\mathrm{j}=1 \\ \mathrm{j} \neq \mathrm{i}}}^{\mathrm{N}} \frac{\mathrm{m}_{\mathrm{j}}}{\mathrm{r}_{\mathrm{ij}}^{\mathrm{a}}} \overrightarrow{\mathrm{r}}_{\mathrm{ij}}$

where $\vec{r}_{i j}=\vec{r}_{j}-\vec{r}_{i}$ and $r_{i j}=\left|\vec{r}_{j}-\vec{r}_{i}\right|$.

A dynamical evolution of the system composed of $N$ - bodies under the influence of gravity is not solvable to exact solution for more than two bodies. Therefore, a numerical approach is required to study this problem. As bodies approach each other, the force between them grows without bound, which is an undesirable situation for numerical integration. In astrophysical simulation, collisions between bodies are generally precluded; this is reasonable if the bodies represent galaxies that may pass right through each other. Therefore, a softening 
factor $\epsilon>0$ is added, and the denominator of eq. (2) is rewritten as follows [10]

$\vec{F}_{i j}=G_{i} \sum_{\substack{j=1 \\ j \neq 1}} \frac{m_{j}}{\left(r_{i j}+\epsilon\right)^{3}} \vec{r}_{i j}$

The softening factor models the interaction between two point masses: masses that behave as if they were spherical galaxies.

In effect, the softening factor limits the magnitude of the force between the bodies, which is desirable for numerical integration of the system state. To integrate over time, we need the acceleration $\overrightarrow{\mathrm{a}}_{\mathrm{i}}=\frac{\overrightarrow{\mathrm{F}}_{\mathrm{i}}}{\mathrm{m}_{\mathrm{i}}}$ to update the position and velocity of body $i$, and so the computation will be simplified to the following form [4]:

$\vec{a}_{i}=G \cdot \sum_{\substack{j=1 \\ j \neq i}}^{N} \frac{m_{j}}{\left(\left(r_{j}-r_{i}\right)^{2}+\epsilon^{2}\right)^{\frac{3}{2}}}\left(\vec{r}_{j}-\vec{r}_{i}\right)$

The integrator used to update the positions and velocities is leapfrog because it is applicable to this problem and is computationally efficient. The choice of integration method in $N$-body problems usually depends on the nature of the system being studied.

\section{Mathematics of MOND}

The flatness of galaxies rotation curves at large radii was the first piece of observational evidence pointing out the possibility that there must be more mass in galaxy than its luminous matter shows. One interesting option is the MOND theory, which is based on a changing of Newton's second law rewritten in the form [11]

$$
\mathrm{m} \mu\left(\frac{\mathrm{a}}{a_{0}}\right) \overrightarrow{\mathrm{a}}=\overrightarrow{\mathrm{F}}
$$

$\mathrm{a}_{\mathrm{o}}$ define a critical acceleration; which below it Newton's second law is not valid anymore. $\mathrm{a}_{\mathrm{o}}$ can take a universal value around $\mathrm{a}_{0} \sim 10^{-}$ $8 \mathrm{~cm} / \mathrm{s}^{2}$. The general form of the MOND theory is given by $\mathrm{m} \mu\left(\frac{a}{a_{o}}\right) \overrightarrow{\mathrm{a}}=\mathrm{G} \frac{\mathrm{Mm}}{\overrightarrow{\mathrm{r}}^{3}} \overrightarrow{\mathrm{r}}$

$\mu(\mathrm{x})$ can take the form $\mu(\mathrm{x})=\frac{\mathrm{x}}{\sqrt{1+\mathrm{x}^{2}}}$.

For technical reasons, it is convenient to rewrite eq. (7) as

$$
\begin{aligned}
& \mathrm{G} \frac{\mathrm{Mm}}{\overrightarrow{\mathrm{r}}^{3}} \overrightarrow{\mathrm{r}}=\mathrm{m} \mu\left(\frac{\mathrm{a}}{a_{o}}\right) \overrightarrow{\mathrm{a}} \\
& \mathrm{G} \underset{\mathrm{r}^{3}}{\frac{\mathrm{M}}{\mathrm{r}}}=\mu\left(\frac{\mathrm{a}}{a_{o}}\right) \overrightarrow{\mathrm{a}}
\end{aligned}
$$

taking into account that $\mathrm{a} \ll \mathrm{a}_{\mathrm{o}}$ therefore

$\mu\left(\frac{a}{a_{o}}\right)=\frac{a}{a_{o}}$ then one get

$\mathrm{G} \frac{\mathrm{M}}{\mathrm{r}^{2}}=\frac{\mathrm{a}^{2}}{a_{0}}$

From which the acceleration's magnitude can be expressed as [12]

$|\vec{a}|=\sqrt{\frac{\mathrm{GM} a_{o}}{\mathrm{r}^{2}}}$

For a circular motion (centrifugal force)

$\overrightarrow{\mathrm{F}}=\mathrm{m} \cdot \overrightarrow{\mathrm{a}}=\mathrm{m} \frac{\mathrm{v}^{2}}{\mathrm{r}^{2}} \overrightarrow{\mathrm{r}} \mathrm{so}|\vec{a}|=\frac{\mathrm{v}^{2}}{\mathrm{r}}$ then

$|\vec{a}|=\frac{\mathrm{v}^{2}}{\mathrm{r}}=\frac{\sqrt{\mathrm{GM} a_{0}}}{\mathrm{r}}$

Finally, the circular velocity is given by [13]

$\mathrm{V}(\mathrm{r})=\sqrt[4]{\mathrm{GM}(\mathrm{r}) a_{o}}$

It can be seen that the circular velocity is independent on the distance $r$ from the center.

\section{Results and Discussion}

Newtonian interaction of simulation governed by the Newtonian law (Newtonian gravity), requires solving $(6 \mathrm{~N})$ ordinary differential equations (three for object position and the other three for velocity) to describe the astronomical object in space. Particle-Particle (P-P) method will be adopted to achieve the desired simulation. Elliptical distribution can be described by two descriptors: the major and minor radius. It is assumed here that the ratio between the major and minor radii is double. To generate 
an elliptical distribution; the polar coordinates will be used to discribe the object location as:

$\mathrm{x}=2 * \mathrm{r} \cos (\theta)$

$y=r \sin (\theta)$

An initial angle $\theta$ is drawn uniformly between 0 and $2 \pi$. The object's velocity is governed by

$\mathrm{v}_{\mathrm{x}}=\mathrm{r}_{\mathrm{p}} * \mathrm{v} * \cos (\theta)$

$\mathrm{v}_{\mathrm{y}}=\mathrm{r}_{\mathrm{p}} * \mathrm{v} * \sin (\theta)$ $\mathrm{v}_{\mathrm{z}}=$ randn

where $r_{p}$ object distance from the center of the simulated distribution and $\mathrm{v}$ is suggested initial velocity, while the mass distribution of the object will be unity but the central mass that will be big enough like $1 \times 10^{5} \mathrm{M}_{\odot}$. Leapfrog numerical techniques were used to solve the $N$-body simulation ODE. To start the simulation the parameters shown in Table 1 were taken.

Table1: Parameters of simulation for elliptical distribution model under Newtonian gravity

\begin{tabular}{|c|c|c|c|c|c|c|c|}
\hline $\begin{array}{c}\text { Number of } \\
\text { star } \\
\text { particles }\end{array}$ & Time step & $\begin{array}{c}\text { Number of } \\
\text { iteration }\end{array}$ & Radius & $\begin{array}{c}\text { Total } \\
\text { mass }\end{array}$ & Velocity & $\begin{array}{c}\text { Velocity } \\
\text { dispersion }\end{array}$ & Shape factor \\
\hline 3000 & $\mathbf{0 . 0 0 0 1 5}$ & 32128 & 5 & $1 \times 10^{5}$ & 30 & random & 2 \\
\hline
\end{tabular}

The output simulations for this configuration are shown Figs. 1 to 4 in 3-D and 2-D coordinates respectively.

From Fig. 1 it's shown that there is essential change in the objects distribution with respect to the previous distribution cases. A spiral galaxy at last has been generated. The two distinct arms are generated after approximately 322 million years. But the problem is that those arms are not stable and vanish after approximately 1.2 billion years.

It seems that the shape factor of the ellipse is the key of spiral shape generation. One can connect this phenomenon to the gravitational force difference acting on the outer part of the initial distribution objects (objects with minor distance distribution affected by larger force than those with major distance). This force (attraction towards the center); and rotation about the center generate arms. This difference in acting force bends the nearest objects inside while the far objects outside the distribution center in which finally generate the spiral shape. But the problem is that those arms are not stable and will vanish after about 1.2 billion years because the gravitational force becomes stronger with time. The change in the shape distribution will affect the shape of rotation curve as in Fig.2. The change in rotation curve behavior will be logically related to the arms formation. The rotation curve is still obeying Keplerian laws; where when these arms dissipated the rotation curve plane restore its usual shape. This behavior is shown clearly in Fig. 3 and 4 where the velocity decreases with distance from the center.

After applying Newtonian theory which didn't output good results; the attention is directed to other hypotheses, which is MOND to test the modification considering the above cases taking into account the modification law of gravitational force (acceleration).

Table 2 lists the parameters that will be used to start the experiment and monitor the behaviour differences. 

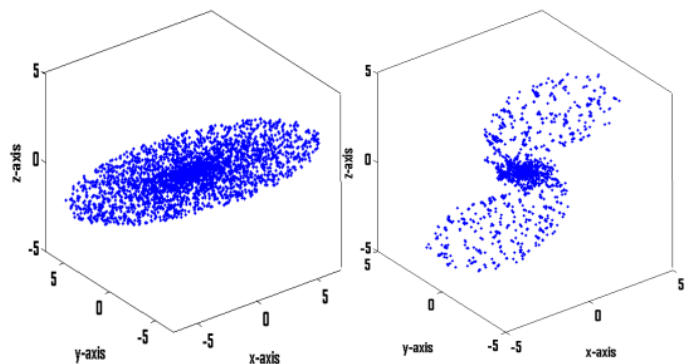

Start

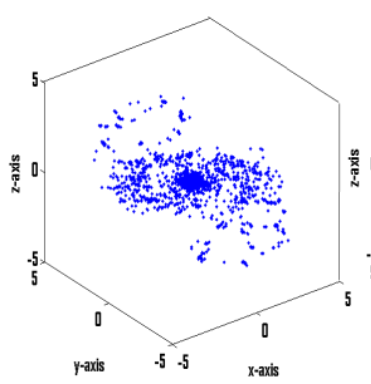

626.28 million years

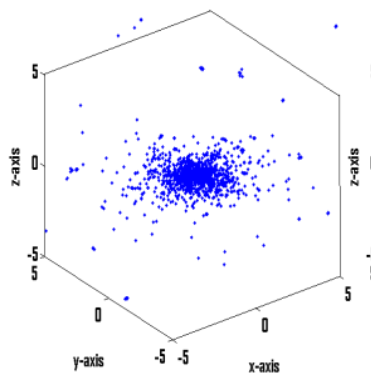

1.272 billion years

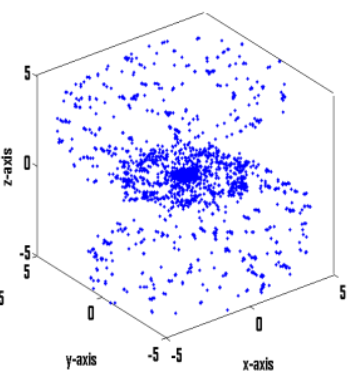

920 million years

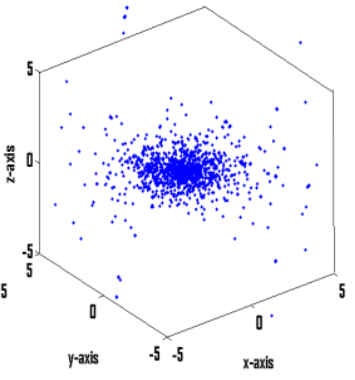

1.572 billion years

Fig.1: A time sequence of the spiral hot disk model in the xyz plane.

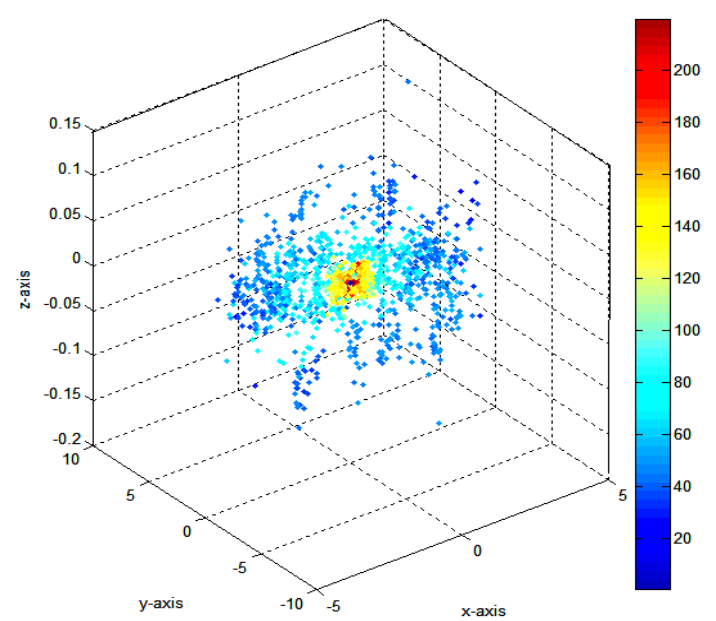

Fig.3: Velocity distributions in xyz plane of the spiral hot disk model after 626.28 million years.
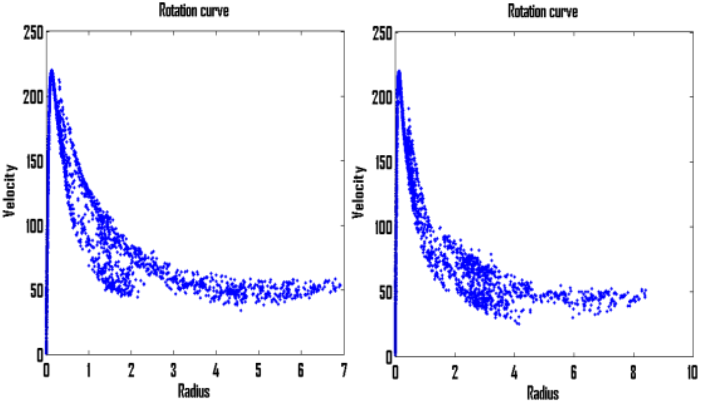

322.9 million years

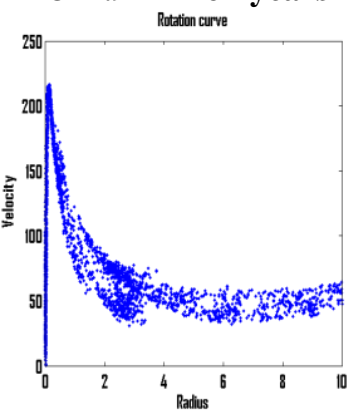

626.28 million years

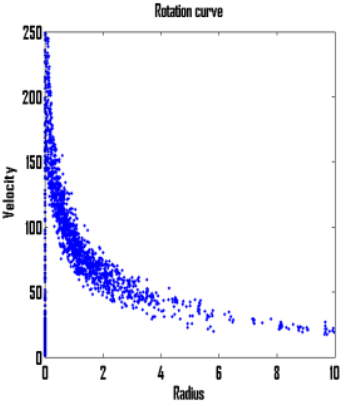

920 million years

1.272 billion years

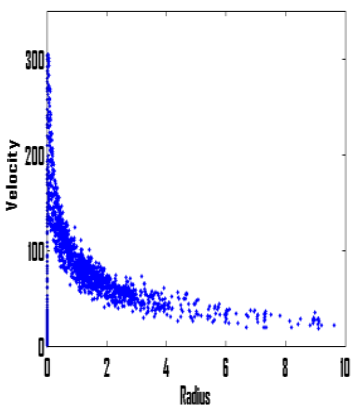

1.572 billion years

Fig.2: Rotation curve of the spiral hot diskmodel aftermany million years.

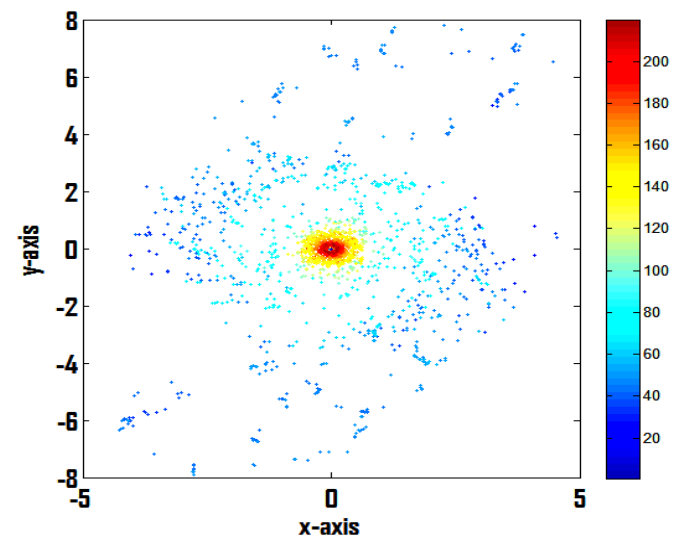

Fig.4: Velocity distributions in $x-y$ plane of the spiral hot disk model after 626.28 million years. 
Table 2: Parameters of simulation for elliptical distribution model under MOND

\begin{tabular}{|c|c|c|c|c|c|c|c|}
\hline $\begin{array}{c}\text { Number } \\
\text { of star } \\
\text { particles }\end{array}$ & $\begin{array}{c}\text { Time } \\
\text { step }\end{array}$ & $\begin{array}{c}\text { Number } \\
\text { of } \\
\text { iteration }\end{array}$ & Radius & $\begin{array}{c}\text { Total } \\
\text { mass }\end{array}$ & Velocity & $\begin{array}{c}\text { Velocity } \\
\text { dispersion }\end{array}$ & $\begin{array}{c}\text { Shape } \\
\text { factor }\end{array}$ \\
\hline 3000 & $\mathbf{0 . 0 0 0 1 5}$ & $\mathbf{8 5 6 7 6}$ & 5 & $1 \times 10^{6}$ & 80 & random & 2 \\
\hline
\end{tabular}

Fig.5 to 8 display the output simulation results of this case.
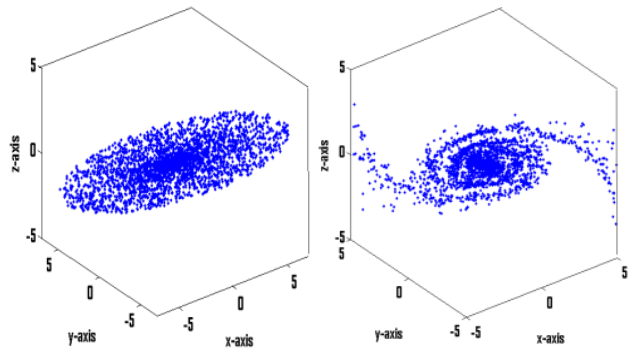

Start

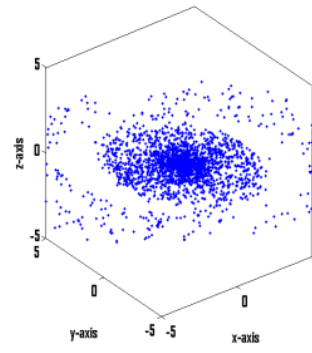

626.28 million year:

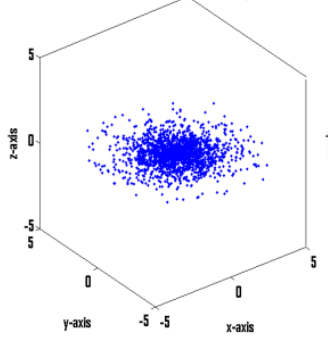

920 million years

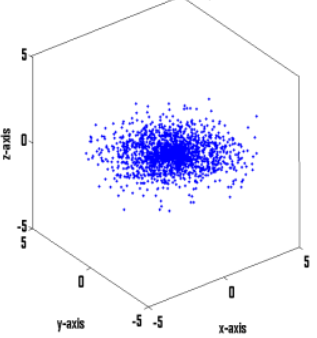

1.272 billion years 1.572 billion years

Fig.5: A time sequence of the spiral hot disk model in the xyz plane.
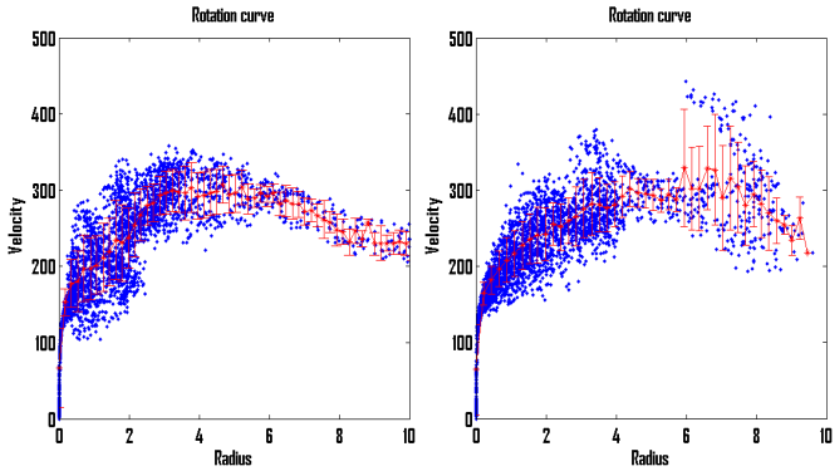

322.9 million years

626.28 million years
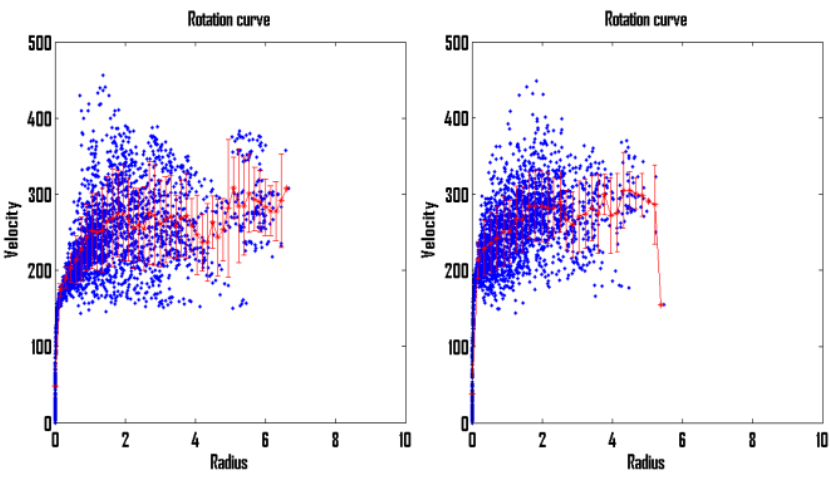

920 million years Rotation curve

1.272 billion years

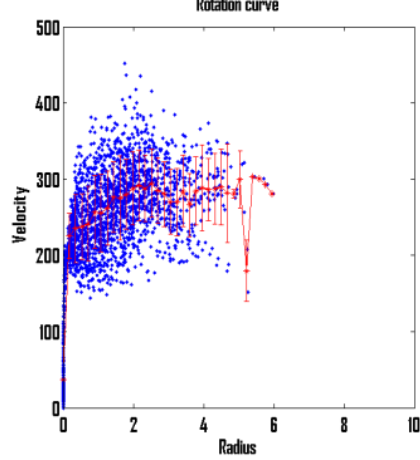

1.572 billion years

Fig.6: Rotation curve of the spiral hot disk model after many million years. 


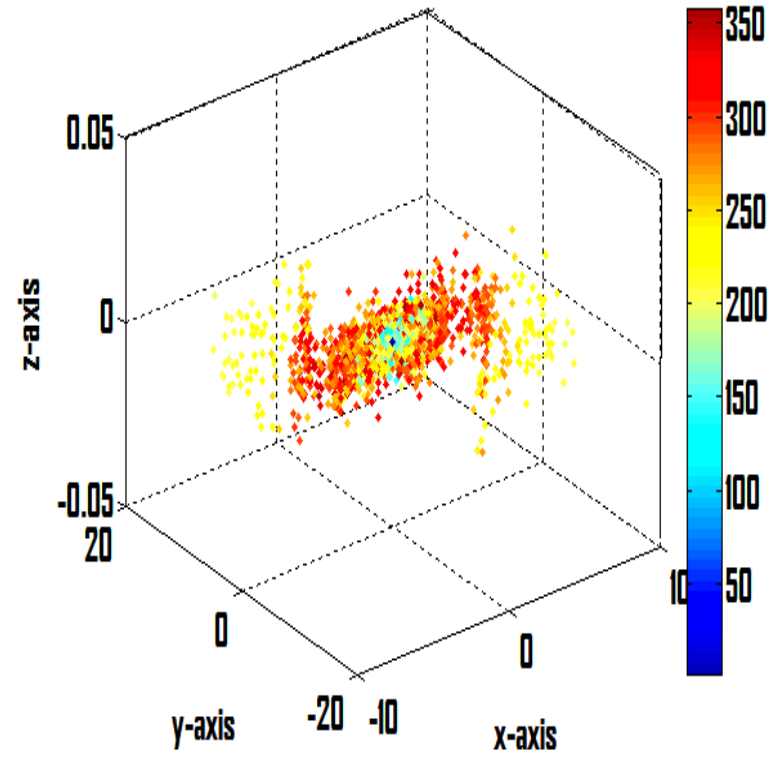

Fig.7: Velocity distributions of the spiral hot disk in space after 322.9 million years.

A very clear and beautiful spiral distribution is gained. Sharper spiral shape and earlier is reached than Newtonian and even longer arms period time is reached (see also Fig.7 and 8 for velocity distribution).

As expected those arms were not stable and finally will merged with the rest of the distribution into a disk-like. The corresponding rotation curve (Fig.6 shows the desired behaviour. This curve is seen to be shrinking with time, where the velocity distribution collapses to the inside according with increasing gravitational acceleration (to compare see reference [14]).

\section{Conclusions}

The proposed modified Newtonian simulations give best results for the distribution and rotation curves for spiral galaxies. It explains most of the galaxy rotation curve without introducing dark matter. Generally, the important result reached in this work is that the modified Newtonian simulation gives the same general behavior for the observed curves of spiral galaxies.

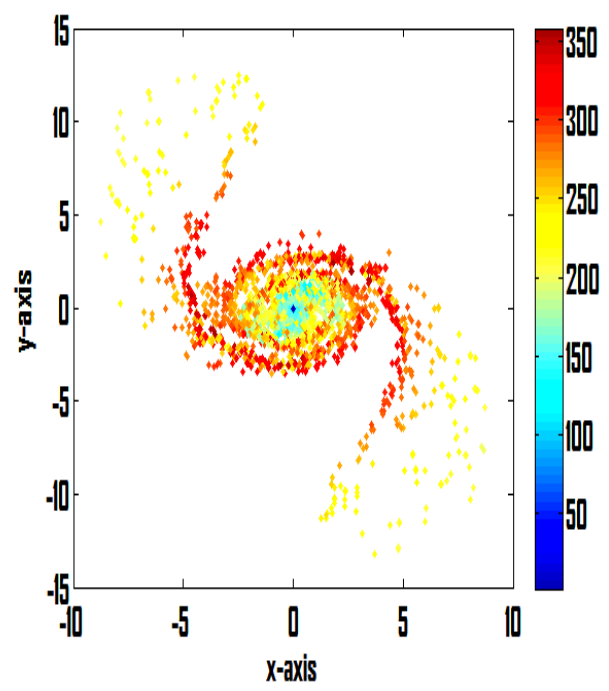

Fig.8: Velocity distributions in $x-y$ plane of the spiral hot disk model after 322.9 million years.

\section{References}

[1] V. Springel, S.D.M White, A. Jenkins, C.S.Frenk, N.Yoshida, L. Gao, J. Navarro, R.Thacker, D.Croton, J.Helly, J.A. Peacock, S.Cole, P. Thomas, H.Couchman, A. Evrard, J.Colberg, and F. Pearce : Nature 435(2005) 629-636.

[2] V.Springel , N.Yoshida and S.D.M. White : New Astron., 6 (2001) 79-117.

[3] J. Dubinski, J. Kim, C. Park and R. Humble : New Astron, 9 (2004) 111-126.

[4] J. Arseth Sverre., "Gravitational N-body simulation" (2003) p2.

[5] V.Springel, Mon. Not. R. Astron. Soc. 364 (2005) 1105-1134.

[6] J.Shenand A.Sellwood, ApJ. 604 (2004) 614.

[7] D. Ceverinoand A. Klypin, MNRAS. 379 (2007) 1087. 
[8] N.Voglis, I. Stavropoulos and C.Kalapotharakos, Mon. Not. R.Astron. Soc. 372 (2006) 901.

[9] M. Martig and F. Bournaud, Draft version April 14 (2010) 20.

[10] D. Dyer, C. Charlesand P. Peter, ApJ. 409 (1993) 60-67.

[11] M. Milgrom, ApJ. 270 (1983) 365-37.
[12] M. Milgrom. ApJ.270 (1983) 371-389.

[13] R. H.Sanders , Advances in Astronomy 752439 (2009) 9.

[14] E.Grivand V. Zhytnikov Vadim, Astrophysics and Space Science, 226 (1995) 51-72. 